\title{
Qualidade do sono e fatores associados em acadêmicos de Medicina: revisão integrativa
}

Sleep quality and associated factors in medical students: integrative review

Calidad del sueño y factores asociados en estudiantes de medicina: revisión integradora

Larissa Daltoé Moreira da CosTA

Curso de Medicina, Faculdade Morgana Potrich, FAMP, 75830-000 Mineiros - GO, Brasil https://orcid.org/0000-0002-7884-8082

Kiane Rayza Pompeu Cunha RODRIGUES

Curso de Medicina, Faculdade Morgana Potrich, FAMP, 75830-000 Mineiros - GO, Brasil https://orcid.org/0000-0003-3026-902X

Neire Moura de GOUVEIA

Curso de Medicina, Faculdade Morgana Potrich, FAMP, 75830-000 Mineiros - GO, Brasil https://orcid.org/0000-0002-2460-4015

\section{Resumo}

Introdução: O sono é classificado como um estado de consciência complementar ao quadro de vigília, contudo, esta revisão traz que em meio ao âmbito acadêmico, no qual ocorrem constantes estresses, os estudantes de medicina são uma classe visivelmente afetada por distúrbios do sono. Estes trazem aos estudantes consequências negativas em seu desempenho acadêmico. Objetivo: $O$ presente trabalho se propõe a fazer uma revisão integrativa para traçar o perfil dos estudantes de graduação em Medicina, quanto a má qualidade do sono e verificar a relação com os principais fatores influenciadores. Métodos: Trata-se de uma revisão integrativa da literatura, na qual foram utilizadas as seguintes palavras-chave "estudante de medicina", "sono" e "depressão" suas correspondentes em inglês. Foram incluídos apenas estudos com alunos do ensino superior do curso de medicina realizados nos últimos 10 anos. Foram incluídos artigos e literatura cinzenta de estudos observacionais, consultados desde o ano de 2000 , sendo que os critérios de exclusão se pautam em estudos experimentais, descritivos e de revisão. Resultados: $100 \%$ dos estudos apresentavam por objetivo dissertar sobre a qualidade do sono e os fatores influenciadores deste especificamente nos estudantes de medicina, assim através dessa revisão percebe-se que a autocobrança realizada pelos estudantes de medicina leva aos distúrbios de sono nestes, sendo que, o uso de substancias/medicamentos são os principais fatores que influenciam diretamente na qualidade do sono dos universitários $32 \%(n=9)$, seguido pela sonolência excessiva diurna $25 \%(n=7)$ e distúrbios psiquiátricos gerais $21 \%$ ( $n=6)$. Considerações finais: Os estudantes de Medicina compõem um grupo susceptível aos transtornos do sono, em razão da carga curricular em horário integral, das atividades extracurriculares e da forte pressão e estresse, com exigência de alto rendimento e tempo demandado em estudos.

Descritores: Sono; Distúrbios do Início e da Manutenção do Sono; Estudantes; Medicina.

\section{Abstract}

Introduction: Sleep is classified as a state of consciousness complementary to the waking state, however, this review brings that in the academic context, which is constantly stressed, medical students are a class visibly affected by sleep disorders. These have the same negative consequences on your academic performance. Objective: The present work proposes to make an integrative review to outline the profile of undergraduate medical students, regarding the poor quality of sleep and to verify the relationship with the main influencing factors. Methods: This is an integrative review of the literature, which used the following keywords "medical student", "sleep" and "depression", their corresponding in English. Only studies with higher education students in the medical course in the last 10 years were included. Articles and gray literature from observational studies, consulted since 2000, were included, and the exclusion criteria are based on experimental, descriptive and review studies. Results: $100 \%$ of the studies aimed to talk about the quality of sleep and its influencing factors specifically in medical students, so through this review it is noticed that the self-charging performed by medical students leads to sleep disorders in these, being whereas, the use of substances / drugs are the main factors that directly influence the quality of sleep of university students $32 \%(n=9)$, followed by excessive daytime sleepiness $25 \%(n=7)$ and general psychiatric disorders $21 \%(n=6)$. Final considerations: Medical students are part of a group susceptible to sleep disorders, due to the full-time curriculum load, extracurricular activities and strong pressure and stress, with high performance and time demanded in studies.

Descriptors: Sleep; Sleep Initiation and Maintenance Disorders; Students; Medicine.

\section{Resumen}

Introducción: El sueño se cataloga como un estado de conciencia que complementa la vigilia, sin embargo, esta revisión muestra que en medio del ambiente académico, en el que hay tensiones constantes, los estudiantes de medicina son una clase visiblemente afectada por trastornos del sueño. Estos tienen consecuencias negativas para su rendimiento académico. Objetivo: Este estudio tiene como objetivo realizar una revisión integradora para delinear el perfil de los estudiantes de medicina de pregrado sobre la mala calidad del sueño y verificar la relación con los principales factores de influencia. Métodos: Se trata de una revisión integradora de la literatura, que utilizó las siguientes palabras clave "estudiante de medicina", "sueño" y "depresión" en sus contrapartes en inglés. Solo se incluyeron los estudios con estudiantes de educación superior de la carrera de medicina realizados en los últimos 10 años. Se incluyeron artículos y literatura gris de estudios observacionales, consultados desde el año 2000, y los criterios de exclusión se basan en estudios experimentales, descriptivos y de revisión. Resultados: El 100\% de los estudios tuvo como objetivo discutir la calidad del sueño y sus factores de influencia específicamente en estudiantes de medicina, por lo que a través de esta revisión se desprende que el autoinforme realizado por estudiantes de medicina conduce a trastornos del sueño en estos, siendo que el uso de sustancias / drogas son los principales factores que influyen directamente en la calidad del sueño de los estudiantes universitarios $32 \%(\mathrm{n}=9$ ), seguidos de la somnolencia diurna excesiva $25 \%(n=7)$ y los trastornos psiquiátricos generales $21 \%(n=6)$. Consideraciones finales: Los estudiantes de medicina son un grupo susceptible a los trastornos del sueño, debido a la carga curricular de tiempo completo, las actividades extraescolares y la fuerte presión y estrés, demandando alto rendimiento y tiempo requerido para los estudios.

Descriptores: Sueño; Trastornos del Inicio y del Mantenimiento del Sueño; Estudiantes; Medicina.

INTRODUÇÃO

O sono é definido como um estado de consciência complementar ao quadro de vigília, a qual envolve mecanismos fisiológicos e comportamentais atuantes em conjunto nas diferentes áreas do sistema nervoso central, este leva à suspensão das atividades voluntárias e sensório-motoras, portanto trata-se de um processo de busca da recuperação do organismo durante o período de vigília. Durante esse processo, vários outros sistemas trabalham juntos para ajudar a manter a homeostase, fixação da memória e regulação da temperatura, sendo que o sono desempenha um papel importante na saúde física e mental de um indivíduo'.

$$
\text { Mudanças no sono podem causar }
$$


comprometimentos cognitivos graves, como dificuldade em fixar e manter a atenção, diminuição da memória, diminuição da capacidade de planejamento estratégico, discinesia e queda do rendimento escolar. Todas essas mudanças costumam levar ao envelhecimento prematuro e reduzir a expectativa de vida do indivíduo ${ }^{1}$.

Como o destaque acadêmico é uma prioridade da maioria dos universitários, estes se submetem a privações de sono durante os dias de aula ou trabalho e são propensos ao uso de substâncias que inibem 0 sono, deixando de lado uma boa higiene do mesmo, é importante ressaltar que atividades sociais e hábitos em geral têm apresentado um desvio para horários cada vez mais noturnos, enquanto as aulas têm iniciado cada vez mais cedo, levando a uma importante redução das horas de sono e um persistente débito de sono durante a semana $^{2,3}$.

Os estudantes de ensino superior apresentam interferências no seu ciclo circadiano sono-vigília mediante o estresse do ambiente acadêmico, o qual é agravado com hábitos como acessar a internet, assistir televisão e fazer uso de álcooll ${ }^{4,5}$. Assim, o presente trabalho se propõe a fazer uma revisão integrativa para traçar o perfil dos estudantes de graduação em Medicina, por meio de estudos primários, quanto a má qualidade do sono e verificar a relação com os principais fatores influenciadores.

MATERIAL E MÉTODO

O presente estudo trata-se de uma revisão integrativa da literatura, o qual seguiu os seguintes passos: definir a população de interesse, definir as palavras chaves, escolha dos critérios de inclusão, busca dos estudos, seleção dos estudos, instrumentos de coletas, obtenção dos dados, análise dos dados, apresentação dos resultados, interpretação dos resultados e obtenção das conclusões. Os dados foram coletados nas bases de dados Literatura Latino-Americana e do Caribe em Ciências da Saúde (LILACS), Scientific Electronic Library Online (SciELO) e States National Library of Medicine (MEDLINE/PubMed) e Biblioteca virtual em saúde Brasil (BVS), no período entre 2010 a 2020. A população de interesse foram "estudantes de graduação do curso de medicina". As palavras-chave utilizadas foram "estudante de medicina", "sono" e "depressão" suas correspondentes em inglês, "medical student", "sleep" and "depression".

Foram incluídos apenas estudos com alunos do ensino superior do curso de medicina realizados no período 2009-2021. Foram critérios de exclusão: artigos publicados antes de 2009 e estudos experimentais, descritivos e de revisão. Somando-se todas as bases de dados, foram encontrados 69 artigos. Porém após a leitura dos títulos dos artigos, notou-se que alguns deles se repetiram nas diferentes bases e outros não preenchiam os critérios deste estudo. Foram selecionados 32 artigos para a leitura do resumo e excluídos os que não diziam respeito ao propósito deste estudo. Após a leitura dos resumos, foram selecionados 21 artigos que preenchiam os critérios inicialmente propostos e que foram lidos na íntegra. $\mathrm{Na}$ seleção final, foram excluídos os artigos de revisão de literatura e relatos de casos. Os artigos escolhidos foram utilizados para a construção de uma tabela comparativa/descritiva dos estudos encontrados e analisados.

RESULTADOS

No que tange ao desenho dos 21 estudos em análise nesta revisão integrativa traz-se que $52 \% \quad(n=11)$ eram do tipo transversal, enquanto que $9 \%(n=2)$ além de transversais eram quantitativos e descritivos. Os artigos randomizados aparecem no percentual de $4 \%(n=1)$, por fim os estudos de revisão foram representados por $33 \%(n=7)$ dos artigos totais revisados como descrito na tabela 1.

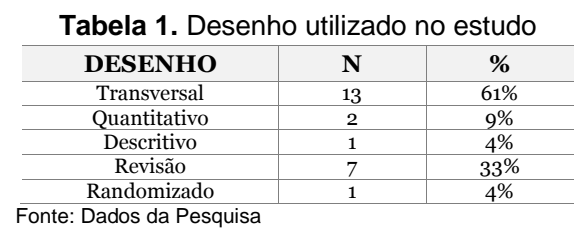

Para obtenção dos resultados os autores fizeram uso em massa da Escala de Sonolência de Epworth (ESE) 42,8\% ( $n=12$ ), e o Índice de qualidade Pittsburgh (IQPS) $39,2 \%(n=11)$ foi o segundo instrumento de coleta mais utilizado pelos autores para avaliação de seus respectivos campos amostrais. Outras escalas foram utilizadas em menores proporções como segue na tabela 2, a justificativa para o maior uso da ESE e IQPS se adentra no fato de ambas terem maiores critérios para estabelecer níveis de insônia e qualidade de sono perante as demais, além de que as mesmas são de fácil aplicação e melhor adesão para o campo amostral.

A insônia nos estudantes de medicina pode ser advinda de fatores diversos, porém a partir dos estudos revisados tem-se que o uso de substancias/medicamentos são os principais fatores que influenciam diretamente na qualidade do sono dos universitários $32 \%(n=9)$, seguido pela sonolência excessiva diurna $25 \%$ 
$(n=7)$ e distúrbios psiquiátricos gerais 21\% $(n=6)$, demais fatores são apresentados na tabela 3 em menores proporções. Entretanto, Pascotto A. \& Santos B. ${ }^{1}$ divergem com todos os demais artigos revisados ao afirmar que a partir de seus dados não se encontra associação entre a sonolência diurna excessiva e a qualidade do sono, sendo que esta somente é afetada a partir de fatores como latência e duração do sono, por este motivo o estudo de ambos não foi posto em contagem na tabela 3 .

Tabela 2. Números de estudos por instrumentos Utilizados

\begin{tabular}{c|c|c}
\hline INSTRUMENTOS & $\mathbf{N}$ & $\mathbf{\%}$ \\
\hline ESE & $\mathbf{1 1}$ & $39 \%$ \\
\hline IQPS & 10 & $35,7 \%$ \\
\hline Questionário Whoqol-bref & 2 & $7,1 \%$ \\
\hline Escala de estresse (PSS-10) & $\mathbf{1}$ & $3,5 \%$ \\
\hline SLEEP-50 & $\mathbf{1}$ & $3,5 \%$ \\
\hline Escala de percepção do estresse (PSS-14) & $\mathbf{1}$ & $3,5 \%$ \\
\hline Escala de Fadiga de Chalder & $\mathbf{1}$ & $3,5 \%$ \\
\hline Fonte: Dados da Pesquisa & &
\end{tabular}

Tabela 3. Fatores influenciadores do sono por estudo

\begin{tabular}{c|c|c}
\hline FATORES & $\mathbf{N}$ & $\mathbf{\%}$ \\
\hline Uso de substâncias/medicamentos & 9 & $32 \%$ \\
\hline Sonolência excessiva diurna & 7 & $25 \%$ \\
\hline Distúrbios psiquiátricos & 6 & $\mathbf{2 1} \%$ \\
\hline Vida sedentária & 3 & $\mathbf{1 0 , 7 \%}$ \\
\hline Insatisfação de rendimento & 3 & $\mathbf{1 0 , 7 \%}$ \\
\hline Dor & 3 & $\mathbf{1 0 , 7 \%}$ \\
\hline Fadiga & 2 & $7,1 \%$ \\
\hline Estresse & 2 & $7,1 \%$ \\
\hline
\end{tabular}

Fonte: Dados da Pesquisa

Apenas $10,7 \% \quad(n=3)$ dos artigos revisados descrevem a média de sono de seus respectivos campos amostrais, sendo que todos convergem em não correlacionar tal média à gênero ou idade. Alsaggaf et al. ${ }^{6}$ trazem que a duração média do ciclo de sono dos participantes da pesquisa realizada varia de 5 a 8 horas e ainda relata que tais costumavam dormir as 1:53 da madrugada. Corrêa et al. $^{3} \mathrm{e}$ Bicho ${ }^{7}$ descrevem médias de sono aproximadas entre seus universitários analisados, porém Corrêa et al. ${ }^{3}$ apontam média é de 6 horas e 59 minutos por noite, enquanto Bicho ${ }^{14}$ destaca que há um período de sono correspondente entre 6 e 7 horas por noite, porém os pacientes demoram cerca de 30 minutos para dormir.

Pascotto e Santos ${ }^{1}$ fazem parte dos $15 \%$ $(n=4)$ de estudos revisados que abordam sobre a influência do gênero no sono e relatam que não são observadas diferenças estatisticamente significantes na qualidade do sono entre os gêneros na descrição da pesquisa de ambos. Enquanto Vaz et al. ${ }^{8}$, Garcia et al. ${ }^{9}$ deixam claro que as mulheres são mais afetadas por distúrbios do sono por apresentarem altos escores de sonolência diurna excessiva, por fim Castro $^{10}$ relata que as mulheres apresentaram qualidade de vida, sono e nível de estresse prejudicados se comparadas aos homens.

Durante a revisão dos artigos pode-se perceber que $100 \% \quad(n=27)$ dos estudos apresentavam objetivo de dissertar sobre a qualidade do sono e os fatores influenciadores deste especificamente nos estudantes de medicina, sendo que em apenas 7,1\% $(n=2) 0$ campo amostral se estendeu a residentes como é descrito na tabela 4, esta traz todos os estudos analisados para construção desta revisão, pois nem todos foram utilizados na discussão e introdução desta por apresentarem objetivos e resultados muito semelhantes, assim através dessa revisão é lúcido que a auto cobrança realizada pelos próprios universitários das faculdades de medicina leva aos distúrbios de sono nestes que traz demais consequências a saúde dos mesmos.

Tabela 4. Informações quanto ao periódico/ano, método, objetivos e resultados dos artigos selecionados

\begin{tabular}{|c|c|c|c|}
\hline $\begin{array}{l}\text { PERIÓDICO/ } \\
\text { ANO }\end{array}$ & MÉTODO & OBJETIVOS & RESULTADOS \\
\hline $\begin{array}{c}\text { Rev Col } \\
\text { Psiquiatr } \\
2019\end{array}$ & $\begin{array}{l}\text { Estudo } \\
\text { transversal }\end{array}$ & $\begin{array}{c}\text { Estimara a } \\
\text { prevalência da } \\
\text { sonolência diurna } \\
\text { excessiva (SDE) e } \\
\text { fatores associados } \\
\text { em estudantes de } \\
\text { medicina de uma } \\
\text { instituição de } \\
\text { ensino superior } \\
\text { (IES) em } \\
\text { Bucaramanga }\end{array}$ & $\begin{array}{l}\text { Os alunos tinham idade média de } \\
20,3 \text { anos; dos } 458 \text { entrevistados, } \\
62,88 \% \text { eram mulheres. Foi } \\
\text { estabelecido que 80,75\% dos } \\
\text { participantes tinham SDE e 80,55\%, } \\
\text { percepção negativa da qualidade do } \\
\text { sono. Na análise multivariada, } \\
\text { constatou-se que o fato de estar } \\
\text { cursando ciências clínicas reduz o } \\
\text { risco de SDE em comparação com } \\
\text { quem cursava o ciclo básico. Além } \\
\text { disso, descobriu-se que uma } \\
\text { pontuação> 15 no IHS aumenta } \\
\text { significativamente o risco de SDE. } \\
\text { Embora comum encontrar SDE em } \\
\text { estudantes de medicina, apenas uma } \\
\text { pequena porcentagem deles sofre da } \\
\text { forma grave desse distúrbio do sono. } \\
\text { O estudo traz a importância de } \\
\text { campanhas que visem melhorar a } \\
\text { percepção de risco do uso de } \\
\text { energéticos por universitários e fazer } \\
\text { recomendações sobre hábitos de } \\
\text { higiene do sono desde o ingresso no } \\
\text { programa acadêmico }\end{array}$ \\
\hline $\begin{array}{c}\text { Braz J } \\
\text { Psychiatry } \\
2019\end{array}$ & $\begin{array}{l}\text { Estudo de } \\
\text { revisão }\end{array}$ & \begin{tabular}{|c|} 
Examinar \\
preditores \\
associados à $(\mathrm{QV})$ \\
em estudantes de \\
medicina \\
brasileiros
\end{tabular} & $\begin{array}{l}\text { Estudantes de medicina do sexo } \\
\text { feminino apresentaram menores } \\
\text { escores de QV nos domínios saúde } \\
\text { física e psicológico do WHOQOL- } \\
\text { Bref em comparação com estudantes } \\
\text { do sexo masculino. Intervenções } \\
\text { específicas devem ser projetadas }\end{array}$ \\
\hline $\begin{array}{c}\text { Rev Bras } \\
\text { Educ Méd } \\
2020\end{array}$ & $\begin{array}{l}\text { Estudo } \\
\text { transversal }\end{array}$ & $\begin{array}{l}\text { O objetivo deste } \\
\text { estudo foi } \\
\text { mensurar os } \\
\text { níveis de fadiga e } \\
\text { sonolência } \\
\text { excessiva diurna } \\
\text { (SED) em } \\
\text { estudantes } \\
\text { internos de um } \\
\text { curso de } \\
\text { medicina, bem } \\
\text { como analisar os } \\
\text { fatores } \\
\text { sociodemográfico } \\
\text { s e pessoais } \\
\text { associados } \\
\end{array}$ & $\begin{array}{l}\text { Aspectos sociodemográficos e } \\
\text { pessoais dos discentes exercem } \\
\text { influência direta sobre os seus níveis } \\
\text { de fadiga e SED. Tal evidência é de } \\
\text { suma relevância, já que fadiga e SED } \\
\text { podem trazer consequências } \\
\text { negativas para os acadêmicos. Um } \\
\text { melhor conhecimento da associação } \\
\text { positiva entre fadiga e SED, bem } \\
\text { como os fatores associados a esses } \\
\text { agravos, permite uma abordagem } \\
\text { dessa problemática por parte das } \\
\text { instituiços de ensino superior, } \\
\text { visando aos melhores desfechos na } \\
\text { qualidade de vida dos discentes e } \\
\text { futuros profissionais médicos }\end{array}$ \\
\hline $\begin{array}{c}\text { Rev Soc Bras } \\
\text { Clin Med } \\
2014\end{array}$ & $\begin{array}{c}\text { Estudo } \\
\text { transversal }\end{array}$ & $\begin{array}{c}\text { Estudar a } \\
\text { qualidade do sono } \\
\text { nos acadêmicos } \\
\text { do curso de } \\
\text { Medicina }\end{array}$ & $\begin{array}{l}\text { Grande parte dos estudantes tem } \\
\text { qualidade ruim de sono e que, } \\
\text { adicionalmente, muitos acadêmicos } \\
\text { com os padrões de sono pobres } \\
\text { referem piora no bem- -estar durante } \\
\text { o dia, como sonolência excessiva } \\
\text { diurna } 5 \text {. }\end{array}$ \\
\hline $\begin{array}{c}\text { J Clin Sleep } \\
\text { Med } 2015\end{array}$ & $\begin{array}{l}\text { Estudo de } \\
\text { revisão }\end{array}$ & $\begin{array}{c}\text { Descrever sobre } \\
\text { os distúrbios do } \\
\text { sono e como este } \\
\text { afeta estudantes } \\
\text { de medicina em } \\
\text { uma perspectiva } \\
\text { global }\end{array}$ & $\begin{array}{l}\text { O sono ruim não é comum apenas } \\
\text { entre estudantes de medicina, mas } \\
\text { sua prevalência também é maior do } \\
\text { que em estudantes não-médicos e a } \\
\text { população em geral. Conclui-se que } \\
\text { há ampla evidência de uma alta } \\
\text { prevalência do problema da insônia } \\
\text { nestes estudantes em especial, e as } \\
\text { pesquisas nesta área devem agora se } \\
\text { expandir para iniciativas que } \\
\text { busquem melhorar a educação geral } \\
\text { do sono para estudantes de medicina } \\
\text { e identificar alunos em risco para } \\
\text { direcioná-los com programas para } \\
\text { melhorar o sono }\end{array}$ \\
\hline
\end{tabular}


Tabela 4 (Continuação). Informações quanto ao periódico/ano, método, objetivos e resultados dos artigos selecionados

\begin{tabular}{|c|c|c|c|}
\hline $\begin{array}{l}\text { PERIÓDICO/ } \\
\text { ANO }\end{array}$ & MÉTODO & OBJETIVOS & RESULTADOS \\
\hline $\begin{array}{c}\text { Rev Bras } \\
\text { Educ Méd } \\
2013\end{array}$ & $\begin{array}{c}\text { Estudo } \\
\text { transversal }\end{array}$ & $\begin{array}{c}\text { Conhecer a } \\
\text { percepção sobre } \\
\text { qualidade de vida } \\
\text { de } 84 \text { estudantes } \\
\text { de graduação em } \\
\text { Medicina da } \\
\text { Universidade de } \\
\text { Brasília }\end{array}$ & $\begin{array}{l}\text { Constatou-se que acadêmicos de } \\
\text { medicina pertencentes a } \\
\text { universidade de Brasília apresenta } \\
\text { uma percepção positiva sobre sua } \\
\text { qualidade de vida, além de que } \\
\text { possuem estrutura suficiente e capaz } \\
\text { de lhes proporcionar boas condições } \\
\text { de vida em um curso que exige } \\
\text { dedicação exclusiva, o que expressa } \\
\text { uma característica marcante e } \\
\text { tradicional da área médica no } \\
\text { Brasil }{ }^{12} \text {. }\end{array}$ \\
\hline $\begin{array}{c}\text { J Bras } \\
\text { Pneumol } \\
2017\end{array}$ & $\begin{array}{c}\text { Estudo } \\
\text { transversal }\end{array}$ & $\begin{array}{l}\text { Avaliar a } \\
\text { percepção } \\
\text { subjetiva da } \\
\text { qualidade do sono } \\
\text { em estudantes de } \\
\text { medicina, } \\
\text { comparando as } \\
\text { diferentes fases } \\
\text { do curso3. }\end{array}$ & $\begin{array}{l}\text { A percepção da má qualidade do sono } \\
\text { mostrou-se elevada para todos os } \\
\text { anos do curso de graduação de } \\
\text { medicina, no entanto, em relação à } \\
\text { comparação das fases do curso, os } \\
\text { alunos dos primeiros anos relataram } \\
\text { pior qualidade do sono e maior } \\
\text { disfunção diurna quando } \\
\text { comparados aos demais3. }\end{array}$ \\
\hline $\begin{array}{c}\text { Saudi } \\
\text { Med J } \\
2016\end{array}$ & $\begin{array}{l}\text { Estudo } \\
\text { transversal }\end{array}$ & $\begin{array}{l}\text { Determinar em } \\
\text { estudantes de } \\
\text { medicina a } \\
\text { qualidade do sono } \\
\text { e associação com } \\
\text { desempenho } \\
\text { acadêmico e } \\
\text { estresse } \\
\text { psicológico }\end{array}$ & $\begin{array}{l}\text { Conclui-se que a má qualidade do } \\
\text { sono e a privação do mesmo é } \\
\text { comum entre os alunos de medicina, } \\
\text { pois os mesmos sofrem com a } \\
\text { constante pressão do curso, que os } \\
\text { fazem manter altos níveis de estresse } \\
\text { diários }^{6} \text {. }\end{array}$ \\
\hline $\begin{array}{l}\text { Rev Bras } \\
\text { Educ Méd } \\
2009\end{array}$ & $\begin{array}{c}\text { Estudo } \\
\text { transversal }\end{array}$ & $\begin{array}{l}\text { Comparar a } \\
\text { qualidade de vida } \\
\text { dos estudantes de } \\
\text { Medicina do } \\
\text { primeiro período } \\
\text { e do último } \\
\text { período do curso }\end{array}$ & $\begin{array}{l}\text { A qualidade de vida dos estudantes } \\
\text { de Medicina, quando avaliada pelo } \\
\text { instrumento Whoqol-bref, sofreu } \\
\text { desgastes no domínio psicológico } \\
\text { durante o curso médico, justificando } \\
\text { novos estudos com desenhos de } \\
\text { Coorte para identificar os fatores que } \\
\text { determinam alterações na qualidade } \\
\text { de vida dos estudantes de Medicina }\end{array}$ \\
\hline $\begin{array}{c}\text { Rev Bras } \\
\text { Educ Méd } \\
2020\end{array}$ & $\begin{array}{c}\text { Estudo } \\
\text { transversal/ } \\
\text { quantitativo }\end{array}$ & $\begin{array}{l}\text { Mensurar os } \\
\text { níveis de fadiga e } \\
\text { SED em } \\
\text { estudantes } \\
\text { internos de um } \\
\text { curso de } \\
\text { Medicina, bem } \\
\text { como analisar os } \\
\text { fatores } \\
\text { sociodemográfico } \\
\text { s e pessoais } \\
\text { associados }\end{array}$ & $\begin{array}{l}\text { Observou-se fadiga em } 85,3 \% \text { dos } \\
\text { internos. Na comparação entre fadiga } \\
\text { e os aspectos sociodemográficos, } \\
\text { constatou-se que a variável sexo } \\
\text { obteve associação significativa, com } \\
\text { maiores escores em alunos do sexo } \\
\text { feminino. Na comparação da fadiga } \\
\text { com os aspectos pessoais, observou- } \\
\text { se associação significativa da fadiga } \\
\text { com os que afirmaram: praticar } \\
\text { atividades físicas raramente, praticar } \\
\text { atividade artística às vezes, fazer } \\
\text { atividade turística às vezes, ter } \\
\text { doença psiquiátrica, ter dificuldades } \\
\text { para dormir, não ser fumante, fazer } \\
\text { uso de substâncias que alteram o } \\
\text { sono e não estar satisfeito com o } \\
\text { próprio rendimento acadêmico. }\end{array}$ \\
\hline $\begin{array}{c}\text { Rev Col } \\
\text { Psiquiatr } \\
2019\end{array}$ & $\begin{array}{l}\text { Estudo } \\
\text { transversal }\end{array}$ & $\begin{array}{l}\text { Implementar } \\
\text { estratégias de } \\
\text { prevenção } \\
\text { primária e } \\
\text { melhorar a } \\
\text { qualidade de vida } \\
\text { dos estudantes }\end{array}$ & $\begin{array}{l}365 \text { alunos obtiveram esportes } \\
\text { indicativos de SDE, com maior } \\
\text { prevalência em mulheres } 236 \text {, O que } \\
\text { nos homens 129. foi observado que } \\
\text { quanto maior o semestre, maiores } \\
\text { eram a escala de gravidade de SDE }\end{array}$ \\
\hline $\begin{array}{c}\text { Rev Bras } \\
\text { Educ Méd } \\
2013\end{array}$ & $\begin{array}{l}\text { Estudo } \\
\text { transversal }\end{array}$ & $\begin{array}{l}\text { Conhecer a } \\
\text { percepção sobre a } \\
\text { qualidade de vida } \\
\text { estudante de } \\
\text { graduação de } \\
\text { medicina da } \\
\text { Universidade de } \\
\text { Brasília }\end{array}$ & $\begin{array}{l}\text { O estudo constatou que estes } \\
\text { acadêmicos apresenta uma } \\
\text { percepção positiva sobre a sua } \\
\text { qualidade de vida, no entanto } \\
\text { reprimi as oportunidades de lazer } \\
\text { destes jovens universitários } \\
\text { conforme apontam os resultados } \\
\text { do estudo. Revela ainda o fato de } \\
\text { que estes possuem estrutura } \\
\text { suficiente para proporcionar boas } \\
\text { condições de qualidade de vida em } \\
\text { um curso que existe dedicação } \\
\text { exclusiva }\end{array}$ \\
\hline $\begin{array}{c}\text { Rev Soc Bras } \\
\text { Clín Méd } \\
2014\end{array}$ & $\begin{array}{c}\text { Estudo } \\
\text { transversal/ } \\
\text { quantitativo/ } \\
\text { descritivo. }\end{array}$ & $\begin{array}{l}\text { Analisar a } \\
\text { qualidade do } \\
\text { sono em } \\
\text { estudantes de } \\
\text { medicina }\end{array}$ & $\begin{array}{l}\text { Dois 184 participantes, a grande } \\
\text { maioria } 148 \text { alunos negaram uso de } \\
\text { substâncias sedativas ou } \\
\text { estimulantes. Entre os que } \\
\text { afirmaram usar tais substâncias, } \\
\text { foi considerável o número de } \\
\text { alunos que os utilizavam mais de } \\
\text { três vezes na semana, totalizando } \\
\text { dez alunos. O tempo habitual para } \\
\text { adormecer variou entre 16 a } 30 \\
\text { min. Correspondendo a } 71 \\
\text { acadêmicos }\end{array}$ \\
\hline $\begin{array}{l}\text { J Clin } \\
\text { Sleep Med } \\
2015\end{array}$ & $\begin{array}{l}\text { Estudo de } \\
\text { revisão }\end{array}$ & $\begin{array}{l}\text { Resumir, } \\
\text { organizar e } \\
\text { esclarecer a } \\
\text { literatura atual } \\
\text { sobre } \\
\text { problemas de } \\
\text { sono entre } \\
\text { graduandos de } \\
\text { medicina em } \\
\text { todo o mundo }\end{array}$ & $\begin{array}{l}\text { Estudantes de medicina são um } \\
\text { subgrupo da população em geral } \\
\text { que parece ser especialmente } \\
\text { vulnerável a sono insatisfatório, } \\
\text { talvez devido à longa duração e alta } \\
\text { intensidade do estudo, tarefas } \\
\text { clínicas que incluem plantões } \\
\text { noturnos, trabalhos que podem ser } \\
\text { emocionalmente desafiadores e } \\
\text { escolhas de estilo de vida }\end{array}$ \\
\hline
\end{tabular}

Tabela 4 (Continuação). Informações quanto ao periódico/ano, método, objetivos e resultados dos artigos selecionados

\begin{tabular}{|c|c|c|c|}
\hline $\begin{array}{l}\text { PERIÓDICO/ } \\
\text { ANO }\end{array}$ & MÉTODO & OBJETIVOS & RESULTADOS \\
\hline $\begin{array}{l}\text { Life Sci } \\
2017\end{array}$ & $\begin{array}{l}\text { Estudo de } \\
\text { revisão }\end{array}$ & $\begin{array}{l}\text { Descrever sobre } \\
\text { associação da } \\
\text { melatonina e } \\
\text { privação do sono }\end{array}$ & $\begin{array}{l}\text { O trabalho em turnos e / ou noturnos } \\
\text { geralmente diminui o tempo de sono } \\
\text { e perturba a estrutura do tempo } \\
\text { circadiano }\end{array}$ \\
\hline $\begin{array}{c}\text { Br J } \\
\text { Pharmacol } \\
2018\end{array}$ & $\begin{array}{l}\text { Estudo de } \\
\text { revisão }\end{array}$ & $\begin{array}{l}\text { Descrever o papel } \\
\text { da melatonina no } \\
\text { sono humano }\end{array}$ & $\begin{array}{l}\text { Como resultado da relação } \\
\text { recentemente descoberta entre } \\
\text { relógio circadiano, o sono e } \\
\text { neurodegeneração, novas } \\
\text { perspectivas de uso da melatonina } \\
\text { para intervenção precoce, para } \\
\text { promover o envelhecimento físico } \\
\text { mental saudável, são de interesse } \\
\text { primordial, tendo em vista a ligação } \\
\text { emergente com a etiologia da doença } \\
\text { de Alzheimer }\end{array}$ \\
\hline $\begin{array}{l}\text { Neurochirurgie } \\
2015\end{array}$ & $\begin{array}{l}\text { Estudo de } \\
\text { revisão }\end{array}$ & $\begin{array}{c}\text { Analisar os efeitos } \\
\text { da melatonina em } \\
\text { humanos }\end{array}$ & $\begin{array}{l}\text { A avaliação da secreção de } \\
\text { melatonina deve ser estendida para } \\
\text { situações em que distúrbios } \\
\text { dramáticos de ritmos endógenos, as } \\
\text { defesas imunológicas e antioxidantes } \\
\text { são patentes, em pacientes } \\
\text { internados para unidades de terapia } \\
\text { intensiva, por exemplo }\end{array}$ \\
\hline $\begin{array}{c}\text { Neurol Res } \\
2017\end{array}$ & $\begin{array}{l}\text { Estudo de } \\
\text { revisão }\end{array}$ & $\begin{array}{c}\text { Fornecer uma } \\
\text { visão abrangente } \\
\text { das várias funções } \\
\text { terapêuticas da } \\
\text { melatonina na } \\
\text { insônia, } \\
\text { distúrbios } \\
\text { respiratórios } \\
\text { relacionados ao } \\
\text { sono, } \\
\text { hipersonolência, } \\
\text { distúrbios sono- } \\
\text { vigília do ritmo } \\
\text { circadiano e } \\
\text { parassonias }\end{array}$ & $\begin{array}{l}\text { A melatonina oferece um tratamento } \\
\text { alternativo às terapias farmacêuticas } \\
\text { atualmente disponíveis prara } \\
\text { distúrbios do sono, com } \\
\begin{array}{l}\text { significativamente menos efeitos } \\
\text { colaterais }\end{array}\end{array}$ \\
\hline $\begin{array}{c}\text { Inquiries } \\
2013\end{array}$ & $\begin{array}{c}\text { Estudo } \\
\text { randomizado }\end{array}$ & $\begin{array}{l}\text { Demonstrar a } \\
\text { relação da } \\
\text { melatonina com } \\
\text { os distúrbios do } \\
\text { sono }\end{array}$ & $\begin{array}{l}\text { O potencial que os agonistas da } \\
\text { melatonina possuem na indústria } \\
\text { farmacológica é considerável. O } \\
\text { desenvolvimento de tais drogas } \\
\text { cronobióticas depende do progresso } \\
\text { no melhor entendimento da fisiologia } \\
\text { do sono e de suas influências } \\
\text { genéticas }\end{array}$ \\
\hline $\begin{array}{l}\text { Rev Col } \\
\text { Bras Cir } \\
2016\end{array}$ & $\begin{array}{c}\text { Estudo } \\
\text { transversal }\end{array}$ & $\begin{array}{l}\text { Avaliar a } \\
\text { qualidade do sono } \\
\text { e a sonolência } \\
\text { diurna de } \\
\text { residentes e } \\
\text { estudantes de } \\
\text { medicina }\end{array}$ & $\begin{array}{l}\text { Participaram cento e cinco residentes } \\
\text { e 101 estudantes de graduação em } \\
\text { medicina. Os } \\
\text { apresentaram média desidentes } \\
\text { superior e pior qualidade do sono } \\
\text { quando comparados } \\
\text { universitários, ambos tiveram } \\
\text { medidas semelhantes de sonolência } \\
\text { pela ESS, mas os residentes } \\
\text { apresentaram menor duração } \\
\text { menor qualidade subjetiva do sono }\end{array}$ \\
\hline
\end{tabular}

\section{DISCUSSÃO}

A qualidade do sono entre estudantes de medicina é um assunto que vem sendo explorado mundialmente devido a suas repercussões silenciosas na rotina acadêmica e na vida pessoal dessa população. Estudos prévios apontam que estudantes com seu tempo total de sono reduzido, têm maior chance de apresentar 0 desempenho acadêmico comprometido ${ }^{3}$.

Em relação, as horas de sono, Segundo et al. $^{6}$ e Almondes e Araújo ${ }^{14}$ corroboram com os dados encontrados, pois correlacionaram-se significativamente a baixa qualidade do sono com dificuldade para dormir, uso de medicamentos para dormir, interferência nas atividades diárias, de acordo com questionários aplicados, os estudantes demoram em média 28,81 minutos para pegar no sono. Além disso, a duração média de sono dos participantes da amostra foi de 5 horas e 50 minutos por noite, tempo inferior à quantidade média de horas 
aceita como normal pelo último consenso sobre insônia, o qual admite como normal 7 a 8 horas por noite ${ }^{15}$.

Instrumentos de pesquisa com análise dos resultados os autores fizeram uso em massa da Escala de Sonolência de Epworth (ESE), seguido da Índice de qualidade Pittsburgh (IQPS) que foi o segundo instrumento de coleta mais utilizado pelos autores para avaliação de seus respectivos campos amostrais. Outras escalas foram utilizadas em menores proporções como segue em tabela Questionário Whoqol-bref , Escala de estresse (PSS-10), SLEEP-50, Escala de percepção do estresse (PSS-14), Escala de Fadiga de Chalder

Os principais fatores que alteram o sono são o uso de substancias/medicamentos, sonolência excessiva diurna e distúrbios psiquiátricos. A qualidade de sono ruim interfere em suas atividades diárias, e estes apresentam dificuldades para adormecer. Com a qualidade de sono afetada, ocorre um aumento de sonolência diurna, algo recorrente entre os estudantes do curso de medicina em especial ${ }^{6}$.

Podemos observar também que as consequências dos distúrbios de sono inicialmente, afetam as variáveis biológicas, as quais trazem consequências imediatas ao organismo, incluindo dor, fadiga, estresse, cansaço, falhas de memória, dificuldade de atenção, e alterações de humor. Em seguida, variáveis funcionais são prejudicadas como, sonolência excessiva diurna, distúrbios psiquiátricos, vida sedentária, insatisfação de rendimento. Por fim, variáveis extensivas são afetadas, sendo observadas em longo prazo, o que consiste em uso de substâncias/medicamentos, sonolência excessiva diurna e surgimento/agravamento de problemas de saúde 6 . Por meio desse estudo, também foi observado diferenças entre gêneros em relação ao grau de sonolência. Isto porque um estudo realizado por uma Faculdade privada de Natal e do Rio Grande do Norte, demonstrou que os homens estudantes no turno noturno obtiveram escore de 9.31 na Escala de Sonolência, e as mulheres, o escore de $9.95^{5,14}$. Vaz et al. ${ }^{8}$ e Garcia et al. ${ }^{9}$ deixam claro que as mulheres são mais afetadas por distúrbios do sono por apresentarem altos escores de sonolência diurna excessiva, por fim Castro $^{10}$ relata que as mulheres apresentaram qualidade de vida, sono e nível de estresse prejudicados se comparadas aos homens. Apesar do sexo feminino ter conquistado o mercado de trabalho em muitas situações elas ainda são as únicas responsáveis por afazeres domésticos e cuidados com os filhos, isso corrobora para um pior desempenho estudantil $\mathrm{e}$, consequentemente, para $\mathrm{o}$ quadro de estresse.

A importância do sono vai além das necessidades fisiológicas, podendo estar associada à alteração do estado geral de saúde dos estudantes, como a sonolência diurna, alterações do humor, confusão mental, diminuição do estado de alerta e danos na memória. É de extrema importância o acompanhamento da saúde do sono desses acadêmicos, para que estes auxiliem em planejamentos de ações de conscientização dessa problemática. Devido à pandemia do COVID19 várias universidades suspenderam as aulas presenciais do semestre, enquanto a avaliação e aulas ocorrem de maneira remota com as aulas on-line. A transição do ensino presencial para o on-line tem um sério impacto na qualidade do sono e fatores estressantes desse grupo. Embora a tecnologia tenha sido usada anteriormente para apoiar o ensino e a aprendizagem, ela também influencia no tempo de tela/internet o que contribui para a insônia nos estudantes de medicina que podem ser advinda de fatores diversos, porém agora atribuída às aulas remotas ${ }^{13}$.

CONSIDERAÇÕES FINAIS

A insônia é um dos distúrbios do sono com grande incidência na população geral, esta influência os ciclos diurnos e noturnos, assim os universitários que são propensos à insônia, devem ser orientados quanto a importância da boa higiene do sono como a prática diária de atividade física, no sentido de reduzir o comportamento sedentário destes, sendo que após esta revisão sistemática afirma-se que a auto cobrança realizada pelos próprios universitários das faculdades de medicina acarreta no surgimento dos distúrbios de sono nos mesmos, pois a maioria dos artigos analisados relatam em seus resultados que o estresse e a ansiedade são grandes fatores etiológicos para desenvolvimento da insônia e tais são sintomas comuns nos universitários de medicina devido à grande carga horária e disciplina exigida pelo curso.

REFERÊNCIAS

1. Pascotto A, Santos B. Avaliação da qualidade do sono em estudantes de ciências da saúde. J Health Sci Inst. 2013 31(3):306-10.

2. Cardoso HC, Bueno FCC, Mata JC, Alves APR, Jochims I, Vaz Filho IHR, Hanna, MM. Avaliação da qualidade do sono em estudantes de Medicina. Rev Bras Educ Méd. 2009 33(3): 349-55. 
3. Corrêa CC, Oliveira FK, Pizzamigli DS, Ortolan EVP, Weber SAT. Qualidade de sono em estudantes de medicina: comparação das diferentes fases do curso. J Bras Pneumol. 2017;43(4):285-89.

4. Solis AC, Lotufo-Neto F. Predictors of quality of life in Brazilian medical students: a systematic review and meta-analysis. Braz J Psychiatry. 2019;41(6):556-67.

5. Ribeiro CRF, Silva YMGP, Oliveira SMC. O impacto da qualidade do sono na formação médica. Rev Soc Bras Clin Med. 2014;12(1): 8-14.

6. Segundo LVG, Cavalcanti Neto, BF, Paz DA, Holanda $M$. Aspectos relacionados à qualidade do sono em estudantes de medicina. Rev Bras Neurol Psiquiatr. 2017;21(3):213-23.

7. Bicho ASS. Estudo da Qualidade do Sono dos Estudantes de Medicina da Universidade da Beira Interior [dissertação]. Portugal: Universidade da Beira Interior; 2013.

8. Vaz ALL, Gléria VOB, Bastos CFC, Sousa IF, Silva AMTC, Almeida RJ. Fatores Associados aos Níveis de Fadiga e Sonolência Excessiva Diurna em Estudantes do Internato de um Curso de Medicina. Rev Bras Educ Méd. 2020; 44(1):1-9.

9. García JAN, Vergel MFB, Labrador JAO, Vera MEO, Olaya HLG. Factores asociados con somnolencia diurna excesiva en estudiantes de Medicina de una institución de educación superior de Bucaramanga. Rev Col Psiquiatr. 2019;48(4):222-31.

10. Castro EJS, Souza DC, Lima PC, Assis IB, Filho ML, Martins FR. Avaliação da qualidade do sono e índice de estresse em acadêmicos de graduação noturna. Núcleo do Conhecimento. 2004;2(4):169-90.

11. Azad MCA, Frasier K, Rumana N, Abdullah AF, Shahana N, Hanly PJ et al. Sleep disturbances among medical students: a global perspective. J Clin Sleep Med. 2015;11(1):69-74.

12. Bampi LNS, Baraldi S, Guilherm D, Araujo MP, Campos ACO. Qualidade de vida de estudantes de Medicina da Universidade de Brasília. Rev Bras Educ Méd. 2013;37(2): 217-25.

13. Rodrigues BB, Cardoso RRJ, Peres CHR, Marques FF. Aprendendo com o Imprevisível: Saúde Mental dos Universitários e Educação Médica na Pandemia de Covid-19. Rev Bras Educ Méd. 2020;44(1):1-5.

14. Almondes K, Araújo J. Padrão do ciclo sonovigília e sua relação com a ansiedade em estudantes universitários. Estudos de Psicologia 2003;8(1):37-43.

15. Poyares D, Rizzo GNV, Minhoto G, Pinto Jr LR, de Souza Bezerra ML, Rodrigues RND et al. I Consenso Brasileiro de Insonia. Hypnos. 2003;4(2):5-39.
16. Touitou Y, Reinberg A, Touitou D. Association between light at night, melatonin secretion, sleep deprivation, and the internal clock: health impacts and mechanisms of circadian disruption. Life Sciences. 2017;173(15):94-106.

17. Nava Z. New perspectives on the role of melatonin inhuman sleep, circadian rhythms and theirregulation. Br J Pharmacol. 2018;175 (16):3190-99.

18. Alsaggaf M, Wali S, Merdad RA, Merdad L. Sleep quantity, quality, and insomnia symptoms of medical students during clinical years. Relationship with stress and academic performance. Saudi Med J. 2016;37(2):173-82.

19. Claustrat B, Leston J. Melatonin: physiological effects in humansla melatonine, role physiologique chez l'homme. Neurochirurgie. 2015;61(2):77-84.

20. Xie Z, Chen F, Li W, Meng X, Feng Y, Liu W et al. A review of sleep disorders and melatonina. Neurol Res. 2017;39(6):559-65.

\section{CONFLITO DE INTERESSES}

Os autores declaram não haver conflitos de interesse

AUTOR PARA CORRESPONDÊNCIA

Neire Moura de Gouveia
Faculdade Morgana Potrich - FAMP
Avenida 3, Q07, Lts 15 a 19, Setor Mundinho
75830-009 Mineiros - GO, Brasil
Email: neiremoura@fampfaculdade.com.br

Submetido em 23/02/2021 Aceito em 16/07/2021 